\title{
Direct Evidence for Curvature-Dependent Surface Tension in Capillary Condensation: Kelvin Equation at Molecular Scale
}

\author{
Seongsoo Kim, ${ }^{1, \dagger}$ Dohyun Kim, ${ }^{1}$ Jongwoo Kim, ${ }^{1,2}$ Sangmin An, ${ }^{1}$ and Wonho Jhe ${ }^{1, *}$ \\ ${ }^{1}$ Center for OD Nanofluidics, Institute of Applied Physics, Department of Physics and Astronomy, \\ Seoul National University, Seoul 08826, Republic of Korea \\ ${ }^{2}$ Center for Convergent Research of Emerging Virus Infection, \\ Korea Research Institute of Chemical Technology, Daejeon 34114, Republic of Korea
}

(Received 11 August 2018; revised manuscript received 20 October 2018; published 12 December 2018)

\begin{abstract}
Capillary condensation is the first-order vapor-to-liquid phase transition taking place in confined geometries. Such heterogeneous nucleation has been well described by thermodynamic laws such as the Kelvin equation, but the equation's applicability at the nanoscale is still unresolved. Here, we show that the Kelvin equation is valid down to approximately $0.5 \mathrm{~nm}$ radius of curvature when the curvature dependence of surface tension is taken into account. By the shear-mode atomic force microscopy, we have measured directly and accurately the critical tip-surface distance $\left(\mathrm{d}_{\mathrm{c}}\right)$ at which the water meniscus is capillary condensed in ambient condition; e.g., $\mathrm{d}_{\mathrm{c}} \approx 1.2 \mathrm{~nm}$ at $10 \%$ relative humidity. In particular, we can determine the Tolman length, the unique characteristic of the curvature-dependent surface tension, as the single fitting parameter $(\delta=0.21 \pm 0.05 \mathrm{~nm})$. Our results that unify the validity of the Kelvin equation at molecular scale and the characterization of the curvature effect of surface tension may provide a better understanding of general nucleation phenomena in nature, including the role of nanometric aerosols in cloud formation.

DOI: 10.1103/PhysRevX.8.041046

Subject Areas: Chemical Physics, Fluid Dynamics, Soft Matter
\end{abstract}

\section{INTRODUCTION}

Understanding how gaseous molecules nucleate is of fundamental importance, but the details are mostly unknown due to the difficulty of directly observing the critical nucleus, the smallest cluster of a new phase [1-4]. Nucleation has generally been described as the thermodynamic processes that consist of two steps: Initial condensation of the critical nucleus by local density fluctuations and subsequent spontaneous growth. Therefore, the critical nucleus represents the very first, but least understood, stage of condensation and provides important information in nucleation theory such as the nucleation rate and activation barrier [5]. However, direct investigation of the critical nucleus is still very challenging at the single nucleus level, and thus only indirect estimation of its characteristics has been made. For example, the critical size of a liquid droplet, being produced by homogeneous nucleation in highly saturated condition due to its "positive" curvature, is very

\footnotetext{
*whjhe@snu.ac.kr

Present address: School of Engineering and Applied Science, Harvard University, Cambridge, Massachusetts 02138, USA.

Published by the American Physical Society under the terms of the Creative Commons Attribution 4.0 International license. Further distribution of this work must maintain attribution to the author(s) and the published article's title, journal citation, and DOI.
}

difficult to be measured directly and thus is inferred indirectly by the nucleation-rate measurement that is possible only after the critical nucleus grows large enough to be counted individually $[5,6]$. On the contrary, capillary condensation of the single meniscus, formed between two surfaces, may provide an ideal platform for direct measurement of the critical nucleus because its "negative" mean curvature allows easy formation and quantitative characterization of the nanoscale meniscus in ambient condition, even at low vapor pressure [4].

Capillary condensation is the readily and ubiquitously observable heterogeneous nucleation in nature [3,7-10]. When two hydrophilic surfaces are nearly in contact, a nano-liquid meniscus is capillary condensed, which plays important roles in, e.g., enhancing adhesion force, increasing (or decreasing) kinetic friction and stimulating cloud formation [8-11]. For its description, the macroscopic Kelvin equation has been employed, which relates the equilibrium curvature of the liquid-vapor interface with the external vapor pressure, as derived by equating the chemical potentials between two bulk phases $[12,13]$;

$$
\frac{1}{R}=\frac{k_{B} T}{v_{l} \sigma} \ln \frac{p}{p_{e}}
$$

where $R$ is the mean radius of curvature such that $1 / R=$ $1 / r_{1}+1 / r_{2}$ for the liquid meniscus [Fig. 1(c)], $k_{B}$ is the Boltzmann constant, $T$ is the temperature, $v_{l}$ is the 

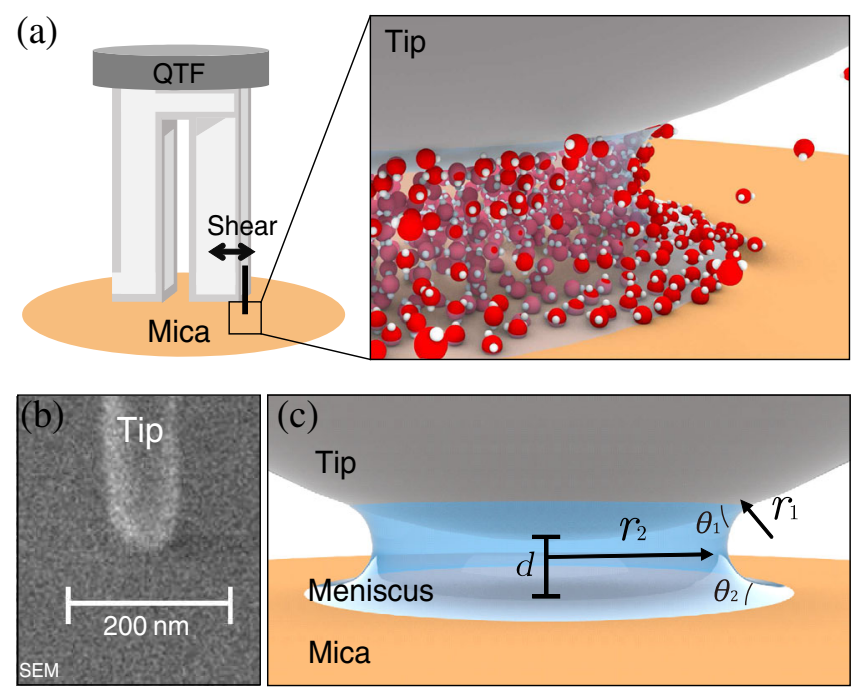

FIG. 1. (a) Experimental scheme of shear-mode quartz-tuningfork-based (QTF-based) AFM. The inset shows the artistic view of the water meniscus having a highly negative curvature at molecular scale. (b) Typical scanning electron microscopic (SEM) image of a quartz tip with radius of about $40 \mathrm{~nm}$ that is glued to one of the QTF prongs. (c) Geometry of the water meniscus. The liquid-vapor interface of the meniscus is in mechanical equilibrium by having the constant mean curvature. Here, $r_{1}$ and $r_{2}$ are the two principal radii-of-curvature $\left(r_{1} \sim-1 \mathrm{~nm}\right.$ is negative and $r_{2} \sim 10 \mathrm{~nm}$ positive in ambient condition) and $d$ is the tip-surface distance. Here, $\theta_{1}$ and $\theta_{2}$ are the contact angles.

molecular volume of liquid, and $p / p_{e}$ is the ratio of external vapor pressure to saturation pressure (i.e., RH value). Notice that $\sigma$ denotes the surface tension, which is assumed to be constant in the Kelvin equation. Since the Kelvin equation is applicable regardless of the presence of a solid [3], it has been established as an equilibrium criterion for homogeneous as well as heterogeneous nucleation [4].

Despite its general and successful use, the validity of the Kelvin equation for the highly curved surfaces still remains as an intriguing problem [13-15]. Previously, the surface-force apparatus experiments on the liquid meniscus showed that the Kelvin equation, which is based on the constant surface tension, is valid down to $|R|$ of $4 \mathrm{~nm}$ for cyclohexane [13] and down to $|R|$ of $5 \mathrm{~nm}$ for water [14]. The relatively large values of $|R|$ are due to the instrumental limits in distance measurement between two surfaces, so the smallest $|R|$ that could be measured is $4-5 \mathrm{~nm}$, which corresponds to about $8-10 \mathrm{~nm}$ distance. Moreover, since water meniscus of $|R|=5 \mathrm{~nm}$ is produced at RH $=90 \%$, higher values of RH beyond $90 \%$ up to saturation have to be employed for stable control of the gap distance above $10 \mathrm{~nm}$, but then the dominant RH-induced systematic effects impose a severe limit on unambiguous discrimination of the pure curvature effect $[13,14]$. Therefore, for the validity study down to the molecular level, one should work at low RH and thus make $|R|$ below $1 \mathrm{~nm}$ (e.g., $|R|=$ $0.23 \mathrm{~nm}$ at $10 \% \mathrm{RH}$ ) while ensuring sub-nm distance control and stable capillary-meniscus formation [16]. In short, the Kelvin equation has not been tested down to $1 \mathrm{~nm}$ using the capillary meniscus until now.

Meanwhile, there has been a longstanding question of whether the surface tension of the flat surface is the same for the highly curved surfaces. From the thermodynamic perspective of surface tension, it was theoretically predicted by Tolman in 1949 that a spherical water droplet has a surface tension that is different from its bulk value when the liquid-vapor interface has a nanoscale radius of curvature of about $1 \mathrm{~nm}$ [17]. Although there have been numerous simulation studies on the Tolman length, which characterizes the curvature dependence of surface tension, the results have been rather different or even contradictory to one another, depending on the theoretical methodologies and the specific water models used [15,18-22]. Despite such extensive theoretical works on homogeneous nucleation, it has been extremely challenging to observe in situ the critical nucleus of a liquid droplet or measure directly its critical size, which has limited a better understanding of the unsettled issue [23]. Hence, the characteristic of the curvature-dependent surface tension has remained as an open question, mostly due to the lack of directly available experimental information on the critical nucleus [5]. Interestingly, the two apparently different topics, test of the Kelvin equation and measurement of the Tolman length, are indeed closely related and thus inseparable from each other, which requires experimental evidence for a mutually consistent and unified understanding.

Here, we demonstrate experimentally that the Kelvin equation is valid down to $|R|$ of $0.5 \mathrm{~nm}$ for water meniscus if and only if the curvature-dependent surface tension is taken into account. We provide the first and direct experimental evidence for curvature dependence of surface tension, which is realized by capturing the very first moment of capillary condensation at a scale of about $1 \mathrm{~nm}$ and measuring accurately the corresponding critical tip-surface distance $\mathrm{d}_{\mathrm{c}}$ at low RH. Importantly, we obtain the Tolman length, which is the unique fitting parameter that bridges between the experiment and the combined Kelvin-Tolman theory. We emphasize that we (i) measured directly the critical size of a purely capillary-condensed (water) meniscus down to $|R|$ of $0.5 \mathrm{~nm}$, (ii) tested the Kelvin equation down to $|R|$ of $0.5 \mathrm{~nm}$, and (iii) determined unambiguously the Tolman length that is derived by the size of the critical nucleus itself. Our results may not only further our understanding of nucleation at a fundamental level but also be useful for various fields such as aerosol physics and material science by similar experiments on other molecules.

\section{EXPERIMENTAL DETAIL AND RESULTS}

\section{A. Experimental scheme}

Highly sensitive, shear-mode, quartz-tuning-fork-based atomic force microscopy (QTF-based AFM) was employed 
$[10,24,25]$. A quartz tip (about $40 \mathrm{~nm}$ radius) was pulled by a mechanical puller (P-2000, Sutter Instrument) and attached to one of the QTF prongs (Fig. 1). The tip approaches the freshly cleaved mica in a humidity-controlled airtight chamber by a precisely calibrated piezoelectric transducer. The tip oscillates laterally at the amplitude of $0.55 \mathrm{~nm}$ and resonance frequency of $32756 \mathrm{~Hz}$. The amplitude-phase response signals are monitored while the tip approaches and retracts at a discrete step of $0.05 \mathrm{~nm}$ displacement and $500 \mathrm{~ms}$ staying time $\left(\tau_{s}\right)$. The tip was retracted immediately after contact in order to prevent any damage to the tip. The full approach up to hard contact was performed only after data taking was completed. We took the SEM image of the quartz tip before and after the $d_{c}$ measurements and found the consistent tip radius of curvature of about $40 \mathrm{~nm}$ (Fig. 9 in Appendix A). The temperature was maintained at $23.3 \pm 0.1{ }^{\circ} \mathrm{C}$, and $\mathrm{RH}$ was controlled at $10 \%, 20 \%$ and $45 \%$ during measurements (accuracy $\pm 2 \%$, Eutech Digi-Sense Thermo-Hygrometer). Before changing RH, we always prepared the freshly cleaved mica and dried the chamber $(\mathrm{RH}<2 \%)$ with dry nitrogen gas. We then set RH at a desired value and left the system equilibrated overnight. We conducted several experiments with different tips and mica samples.

Our measurement system based on QTF-AFM exhibits three unique features that are critical to the present work: (i) high quality factor $\left(Q \sim 5 \times 10^{3}\right)$ and high sensitivity of QTF in ambient conditions to detect precisely mechanical properties of single meniscus [10], (ii) high stiffness $\left(k \sim 10^{3} \mathrm{~N} \mathrm{~m}^{-1}\right)$ to produce stable formation and atomicresolution height control of capillary-condensed meniscus without "jump-to-contact" mechanical instability [14,26], and (iii) shear-mode operation to realize subnanometerresolution distance measurement and accurate determination of the equilibrium curvature by small-amplitude oscillation of the AFM tip.

\section{B. Accurate measurement of $d_{c}$}

We perform direct measurements of the critical tipsurface distance $\left(\mathrm{d}_{\mathrm{c}}\right)$ where capillary condensation occurs, instead of the indirect force measurements [26,27]. The sharp AFM tip experiences condensation, contact, and hard contact during a step-wise full approach to the mica surface [Fig. 2(a)]. The typical approach curve at $10 \% \mathrm{RH}$ and room temperature is presented in Fig. 2(b). A sudden drop of the tip's shear amplitude at $d_{c}$ [position (ii), black curve] implies spontaneous formation of water meniscus [10]. The contact point $(d=0)$ is determined by the dynamic-force spectroscopy method $[24,28]$ for the full-approach curve, where the amplitude-phase signals change rapidly at position (iii) (see Sec. C). Notice that hard contact corresponds to the point where the dissipated energy $E_{\text {dis }}$ is maximized [position (iv), red curve]. Figure 2(c) shows details of the amplitude-phase response during approach and retraction, where we find $\mathrm{d}_{\mathrm{c}} \approx 1.2 \mathrm{~nm}$ at $10 \% \mathrm{RH}$.
Figure 2(d) plots the approach curves at differing values of $\mathrm{RH}(10 \%, 20 \%$ and $45 \%)$, and the corresponding histograms of $\mathrm{d}_{\mathrm{c}}$, obtained by independent 100-times repeated measurements, are presented in Fig. 2(e). Here $\mathrm{d}_{\mathrm{c}}$ was measured as $1.2 \pm 0.08 \mathrm{~nm}, 1.5 \pm 0.1 \mathrm{~nm}$ and $2.2 \pm 0.15 \mathrm{~nm}$, respectively, where the error denotes the standard deviation. The experiment under 2\% RH $\left(\mathrm{d}_{\mathrm{c}} \approx 1 \mathrm{~nm}\right)$ was also conducted but not included in our data analysis because of (i) experimental limits in maintaining such a low RH for a long enough time to make statistical measurements and (ii) the instrumental limit of the hygrometer in measuring the low $\mathrm{RH}$ values below $2 \%$. Between $0 \%$ and $2 \% \mathrm{RH}$, we indeed measured $\mathrm{d}_{\mathrm{c}}$ within the range between $0.7 \mathrm{~nm}$ and $1 \mathrm{~nm}$ (note that $\mathrm{d}_{\mathrm{c}}$ of $1 \mathrm{~nm}$ corresponds to about $-3 \mathrm{~nm}^{-1}$ curvature), which agrees well with our final conclusion (the Kelvin-Tolman equation in Fig. 8). The details of $d_{c}$ measurements for various RHs are presented in Appendix B.

Notice that the Kelvin equation predicts $\mathrm{d}_{\mathrm{c}}$ of $0.6 \mathrm{~nm}$, $0.9 \mathrm{~nm}$, and $1.8 \mathrm{~nm}$ at $10 \%, 20 \%$ and $45 \% \mathrm{RH}$, respectively. In other words, the measured $\mathrm{d}_{\mathrm{c}}$ at each $\mathrm{RH}$ is consistently larger than the theoretical prediction by the Kelvin equation, which assumes constant surface tension. The discrepancies with respect to experiments are especially prominent at low RH: $84 \%$ difference at $10 \%$ RH and $66 \%$ difference at $20 \% \mathrm{RH}$. Nonetheless, we emphasize that, to confirm such apparent deviation from the Kelvin equation, other possible systematic effects as well as statistical analysis should be considered in detail so that our microscopic distance measurements can be validated (see discussions in Secs. II D-II F).

\section{Determination of contact point}

It is very essential to determine the exact contact point, defined by $d=0$ in Fig. 3(a), in order to accurately measure the critical distance. In addition to the inevitable experimental error due to the elasticity of the quartz tip a nd the mica substrate, there exists the ambiguity of the meaning of "contact" at molecular scale. In Fig. 3(a), we show that the values of $d_{c}$ obtained by our measurement are always larger than those estimated by the Kelvin equation, regardless of how the contact point is defined within experimental bounds, so the apparent discrepancy observed between the Kelvin equation and the experiment is evident especially at low RH (see Appendix B).

Another complementary way to define the contact point is by referring to the elastic $\left(F_{k}\right)$ and viscous $\left(F_{b}\right)$ forces of shear interaction that can be calculated by dynamic force spectroscopy [Fig. 3(b)]. When the shear amplitude and phase of the tip change noticeably at the contact point during its full approach, the force acting on the tip also increases very rapidly [24]. A further decrease in amplitude beyond the contact point is mainly due to the elasticity of the tip and the strong repulsive force until hard contact. Notice that the elastic force between two surfaces 
(a)

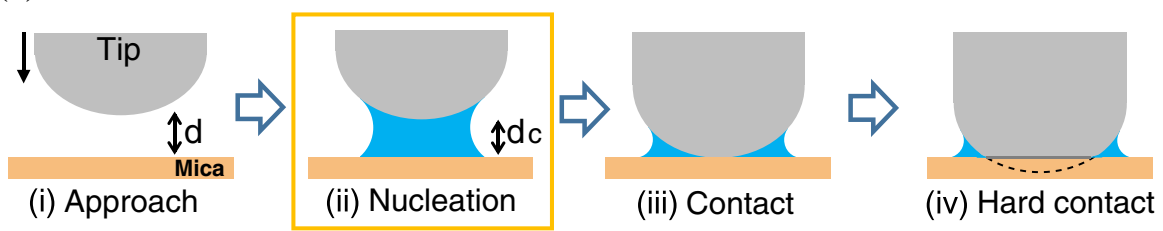

(b)

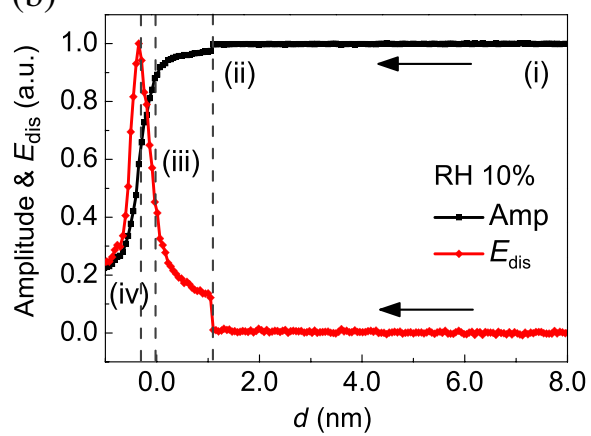

(d)

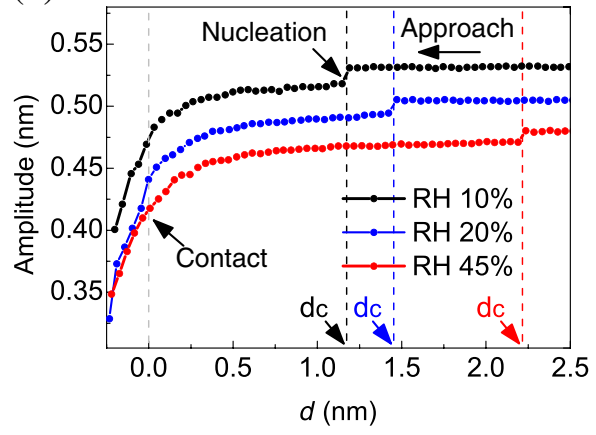

(c)

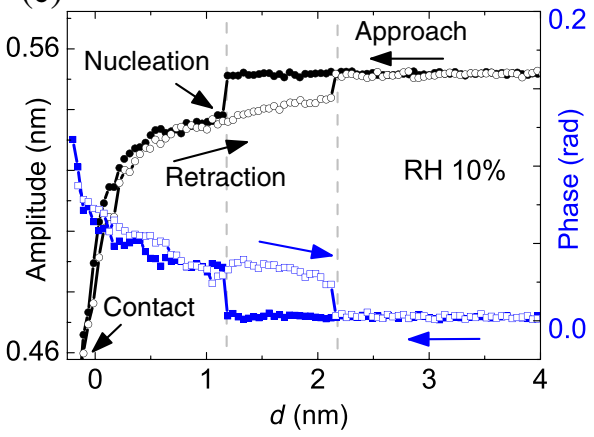

(e)

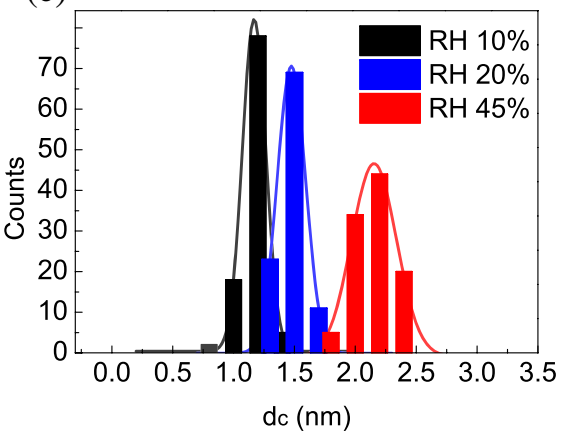

FIG. 2. (a) Schematic of determining $d_{c}$ and the contact point during tip approach. (b) Change of oscillation amplitude and energy dissipation in arbitrary units during the full approach until hard contact. The specific points (i)-(iv) correspond to each step in (a). (c) Typical approach-retraction curve at 10\% RH. Filled black (blue) dots represent the amplitude (phase) change during approach, while the open black (blue) dots show those obtained during retraction. (d) Approach curves versus RH. (e) Histogram plot of $\mathrm{d}_{\mathrm{c}}$ versus RH for 100-times approach-retraction curves. Here, $\mathrm{d}_{\mathrm{c}}$ was measured as $1.2 \pm 0.08 \mathrm{~nm}, 1.5 \pm 0.1 \mathrm{~nm}$ and $2.2 \pm 0.15 \mathrm{~nm}$ at RH of $10 \%, 20 \%$ and $45 \%$, respectively (here, the error denotes the standard deviation).

$(>100 \mathrm{nN})$ is dominant compared to the capillary force (order of 1-10 $\mathrm{nN}$ [27]) when both surfaces come into contact. As the QTF is highly damped beyond hard contact, the amplitude curve shows saturation characteristics, and the associated energy dissipation becomes maximum.

\section{Adsorbed water film}

Let us now address the coalescence effect due to the molecular liquid film of water $(\approx 0.37 \mathrm{~nm}$ thick and $100 \mathrm{~nm}$ wide below $50 \% \mathrm{RH}$ ) that is pre-adsorbed on the freshly cleaved mica at finite RH [29,30], which obviously affects the measured $d_{c}$ values as in Fig. 4(a). We indeed observe such a systematic RH-dependent increase of $d_{c}$. Figure $4(b)$ shows that the presence of liquid film on the mica produces an approximately $0.4-\mathrm{nm}$ shift of $\mathrm{d}_{\mathrm{c}}$ at $20 \% \mathrm{RH}$, unlike the pure nucleation case [4]. Note that while we obtain the shift of $0.45 \mathrm{~nm}$ at $45 \% \mathrm{RH}$ [Fig. 4(c)], we do not find any systematic shift of $d_{c}$ below $10 \%$ RH in separate measurements. Such a RH-dependent increase is indeed already indicated in Fig. 2(e), where the uncertainty of the $d_{c}$ measurement increases with $\mathrm{RH}$, which originates from the pre-adsorbed liquid film. This kind of systematic shift of $d_{c}$ can occur because the muscovite mica is hydrophilic with near-zero contact angle, so scattered molecular water clusters can be produced on the mica, which become thicker and wider at higher RH.

More specifically, the fixed difference $(\approx 0.4 \mathrm{~nm})$ between two values of $d_{c}$ in Figs. 4(b) and 4(c), obtained by several independent sets of measurements made in various conditions (including differing values of $\mathrm{RH}$ ), is indicative of the well-established presence of the preadsorbed water-monolayer clusters $(0.37 \mathrm{~nm}$ thick $)$ at RH less than 50\% [Fig. 4(a)] [30]. It has also been known in the force measurements that the presence of a liquid film simply increases the measured distance by the film 

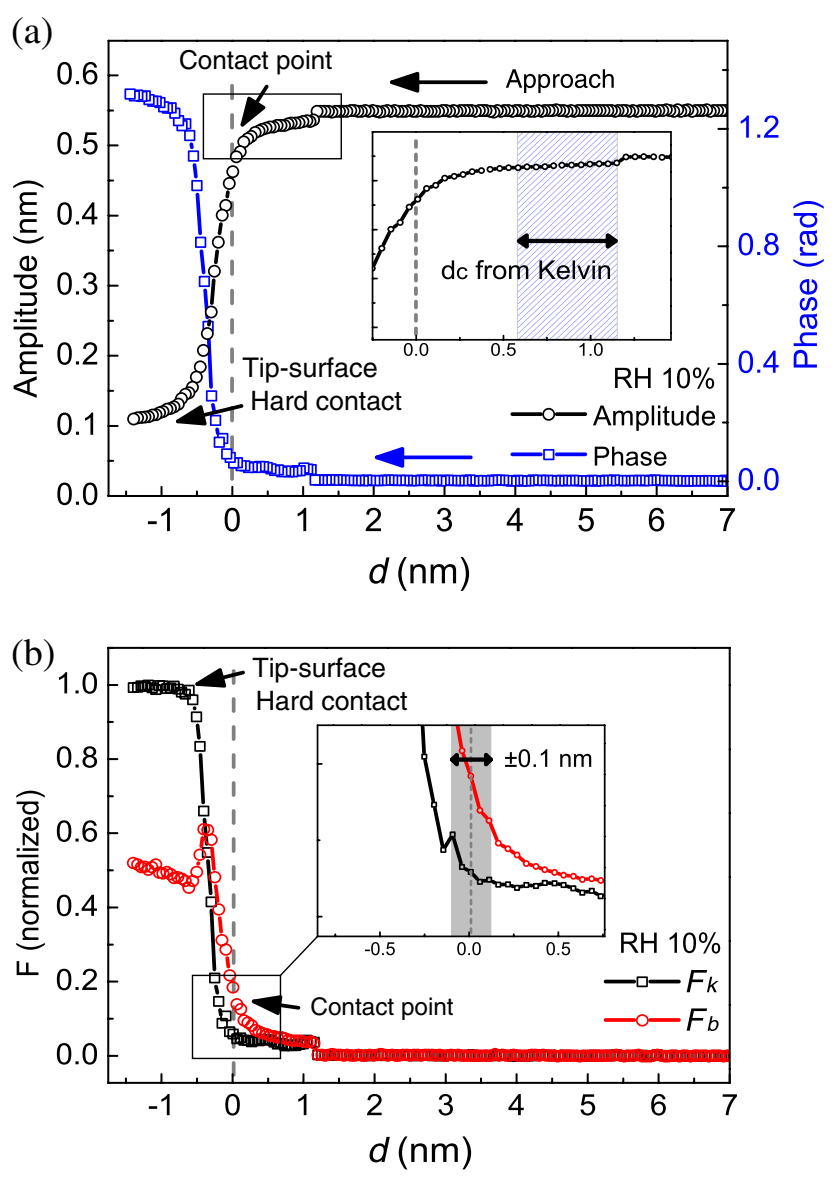

FIG. 3. (a) The tip-surface contact $(d=0)$ is determined as the point where a rapid decrease of the tip's shear amplitude starts during approach. The inset shows that the critical distance calculated by the Kelvin equation (blue shaded region) is still much shorter than the shortest experimental value of $d_{c}$, obtained at $10 \% \mathrm{RH}$, even if the experimental error of contact-point determination $[ \pm 0.1 \mathrm{~nm}$; see inset of (b)] is included. (b) The elastic and viscous forces derived by the corresponding amplitude-phase information [24], where the contact point can be equally determined.

thickness [13], where the radius of curvature of the meniscus was calculated by subtracting the film thickness from the separation between two mica sheets. Therefore, in our analysis to compare with the Kelvin equation, we have used only those data sets of $d_{c}$ that belong to the shorter average values in order to ensure the absence of the liquid film-induced coalescence effect, so only the pure capillarycondensation processes can be analyzed.

\section{E. Surface contamination}

Contamination is an important issue in capillary condensation experiment because any adventitious material can affect the nucleation and alter the equilibrium radius of curvature [14]. In this regard, it has been reported and confirmed that the cleanest mica surface is produced by the tape-cleaving method compared with any other available methods such as hot-wire cutting and rinsing with dilute acid [31]. We have prepared the mica surface following the tape-cleaving method. In addition, in order to check other possible contaminants such as organic particles, we scanned the mica surface that is prepared in the same manner as the one used in our experiment, using a noncontact mode commercial AFM (NX-10, Park Systems) and a commercial cantilever tip ( $2 \mathrm{~nm}$ radius of curvature; SSS-NCLR-10, Nanosensors Co.). We randomly scanned different parts of the cleaved mica surface, and the corresponding typical data are shown in Fig. 5. As shown, we have found less than one contaminant particle among the total $1000 \mu \mathrm{m}^{2}$ scanned area.

Moreover, the small effective contact area between the tip and the mica surface suggests even less vulnerability to the contamination problem. The width of the nanomeniscus is very small due to the small radius of the AFM tip; e.g., the value of $r_{2}$ in Fig. 1 is of the order of 1 to $10 \mathrm{~nm}$, for which the concentration of adventitious particles is practically negligible. We have also performed statistical analysis of $d_{c}$ by approaching the mica surface over 100 times at different locations to confirm the reproducible measurements of $d_{c}$, regardless of the location of the tip. We have also performed several experiments after rinsing the mica with acid solution (Appendix C). We concluded that the tape-cleaving method is the most effective way to prepare the clean mica surface according to the relevant literature $[14,31]$, as also confirmed by our direct scanning of the mica surfaces with a commercial AFM and a well-characterized commercial tip as well as by the indirect evidence of the increased values and uncertainties of the measured $d_{c}$.

\section{F. Systematic effects}

The measured $d_{c}$ values are consistently larger than those predicted by the Kelvin equation that assumes constant surface tension. This indicates that the curvature dependency of surface tension becomes appreciable because of the highly curved interface of the nanomeniscus. However, before fitting with the Kelvin-Tolman equation, other parameters in the Kelvin equation should be characterized in detail because, in such molecular regimes, physical quantities may also deviate from the bulk values just like the surface tension. In this section, we discuss each parameter carefully and show that, even if the uncertainty associated with each quantity is considered, the curvaturedependent surface tension has to be incorporated to fully account for the large discrepancy from the Kelvin equation that deviates as much as $84 \%$ from the experimental values.

\section{Effect of nonzero contact angle}

As the tip oscillates laterally, water molecules also oscillate accordingly, and thus the physical parameters depicted in Fig. 1(c), such as the contact angles $\theta_{1}$ and $\theta_{2}$, may change from the ideal static case. However, such a molecular motion of water would manifest itself, on 
(a)

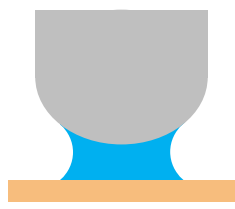

(i) Nucleation on mica

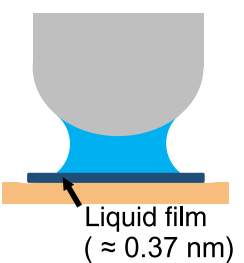

(ii) Coalescence with liquid film (b)

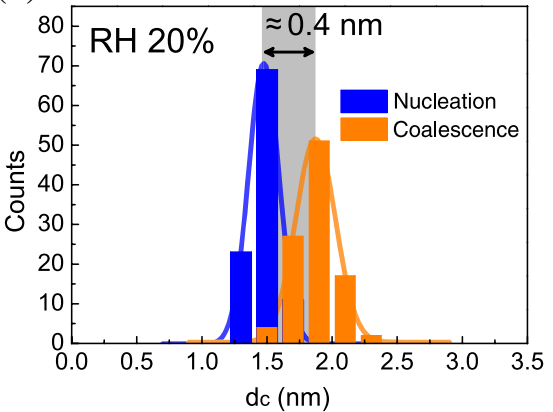

(c)

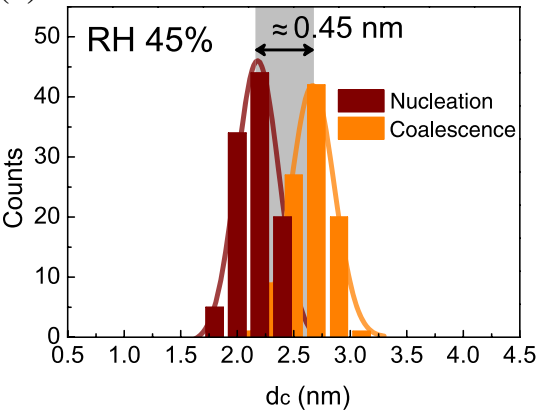

FIG. 4. (a) Liquid film is adsorbed on the mica with about $0.37 \mathrm{~nm}$ thickness (monolayer of water) and $100 \mathrm{~nm}$ width at RH below $50 \%$ $[29,30]$. We performed numerous approach-retraction processes at various $\mathrm{RH}$ and found there are two distinct values of $\mathrm{d}_{\mathrm{c}}$, separated by the liquid-film thickness when RH $\geq 20 \%$. (b) At $20 \% \mathrm{RH}, \mathrm{d}_{\mathrm{c}}$ is either $1.5 \pm 0.1 \mathrm{~nm}$ or $1.9 \pm 0.16 \mathrm{~nm}$ depending on the local presence of liquid film. (c) At $45 \% \mathrm{RH}$, two differing $\mathrm{d}_{\mathrm{c}}$ values are $2.18 \pm 0.15 \mathrm{~nm}$ and $2.63 \pm 0.2 \mathrm{~nm}$. We performed 100 approaches in each case for statistical analysis. The contribution of coalescence due to the liquid film systematically increases the measured critical distance by the thickness of the water monolayer. Our data analysis considers only those results corresponding to pure nucleation.

average, to the measured values. This is because water molecules typically have relaxation time of about ps (for nanoconfined liquid, relaxation time is as long as about $1 \mu \mathrm{s}$ [25]), while the oscillation period is much longer (about $30 \mu \mathrm{s}$ ). In addition, the width of water meniscus is of the order of about $10 \mathrm{~nm}$, while the lateral amplitude of the tip is only about $0.5 \mathrm{~nm}$; thus, the oscillation exerts almost negligible perturbation to the meniscus.

In fact, the issue can be further extended to whether one can actually define the contact angle, which is ultimately related to the question of the applicability of thermodynamics at molecular level. A recent simulation study indicates that the temporal averaging of inhomogeneity coming from the discontinuous nature of matter allows one

(a)

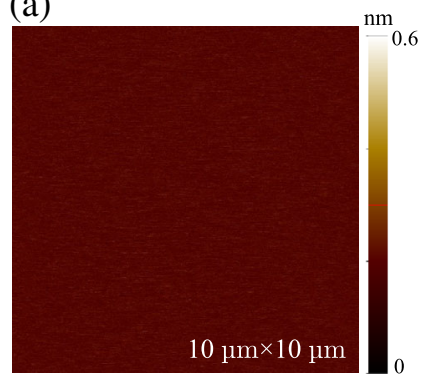

(b)

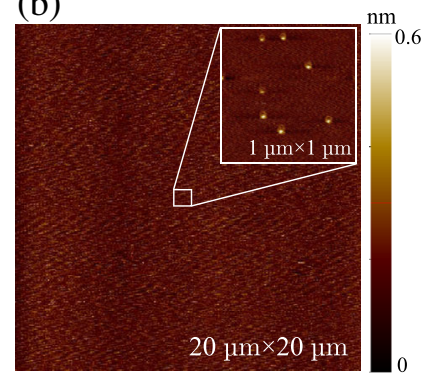

FIG. 5. (a) Mica surface scanned by a noncontact mode commercial AFM (NX-10, Park Systems) with a commercial cantilever tip ( $2 \mathrm{~nm}$ radius of curvature; SSS-NCLR-10, Nanosensors Co.). The mica surface was equilibrated in an airtight chamber for over one day, which is the same condition as we used for our capillary condensation experiments in this paper. After randomly scanning different areas on the surface, we found that no contaminants exist over a $1000 \mu \mathrm{m}^{2}$ scan area. Mica was prepared using the tape-cleaving method, which is well known to produce the cleanest mica surface [31]. (b) Mica surface that was placed in airtight chamber for over 14 days. We found high concentration of contaminants scattered over the mica surface. The inset clearly shows adventitious particles. to use and apply thermodynamics to the molecular level with considerable accuracy [15]. Moreover, in the present work, even if we assume that the contact angle between the meniscus and the quartz tip takes a value between 0 and 30 degrees, such a variation only has a minor contribution to the experimental error in $d_{c}$-less than $15 \%$ difference between 0 and 30 degrees. Furthermore, the contact-angle difference would make even larger discrepancies between the experimental $d_{c}$ and the one from the Kelvin equation, so our conclusion still holds.

\section{Effect of nonideal fluidity}

Assumptions of ideal gas law and incompressible liquid used to derive the Kelvin equation should be examined, as our large $d_{c}$ measurements might merely originate from the nonideal fluidity. Melrose investigated a correction to the Kelvin equation based on the density dependence of the isothermal thermodynamic susceptibility [32]. He concluded that the major correction to the Kelvin equation from nonideal fluidity is from the dilation in the liquid by a strong negative pressure. It is estimated that the fractional increase in molar volume of the liquid can be $17 \%$. This will produce at most $34 \%$ larger critical distance $\left(d_{c} \sim 2\left|r_{1}\right|\right)$, which is still insufficient to explain our large $d_{c}$ measured at low RH (84\% difference at 10\% RH and 66\% difference at $20 \% \mathrm{RH}$ ).

\section{Effect of bulk volume density}

In our analysis, we assume the bulk volume density for the molecular volume $v_{l}$ in Eq. (1). Although it is widely known that water molecules are more structured near a solid surface, theoretical [33] and experimental works [34] support the fact that the water density near the solid surface has little deviation from the bulk value. Grand canonical Monte Carlo simulation predicts little difference on the density of the condensed phase compared to the bulk 
value [33], which is in accordance with the homogeneous nucleation experiments [23]. Furthermore, the surface force apparatus experiment shows that the meniscus formed by capillary condensation has a refractive index similar to the bulk value until its rupture [34]. Nonetheless, we note that further research on how the structured molecules near the solid surface affect the nucleation as well as the liquidvapor interface would significantly improve the precision of the Tolman length measurement.

\section{Effect of surface roughness}

Another possible source of systematic error could be the surface roughness, which is important in many wetting phenomenon and should be checked thoroughly. We scanned the mica and the quartz tip using a commercial AFM (Park Systems, NX-10) with a commercial cantilever tip (2-nm radius of curvature; SSS-NCLR-10, Nanosensors Co.). We found that the mica surface is atomically flat as expected. The measured root-mean-square (rms) surface roughness of mica is $0.2 \AA$, which is much smaller than the typical value of $\mathrm{d}_{c}(\sim 1 \mathrm{~nm})$. On the other hand, since it is extremely difficult to scan the very apex of the quartz tip for the roughness measurement, we scanned the side surface of the tip that is expected to have a roughness similar to the tip apex. The scanned image of the quartz tip is shown in Fig. 6. The measured rms roughness of quartz is $0.1 \mathrm{~nm}$. We attribute the roughness value of quartz, which is small compared to the one reported in other literature [35], to the smaller scanning area and the elasticity of quartz.

We also qualitatively confirm the smooth tip apex by the SEM image (Fig. 1). Moreover, in our $d_{c}$ measurements, we have not observed any signal fluctuations when the tip contacts the mica substrate or when the water meniscus is ruptured, which is the characteristic of the signal obtained by a smooth tip. In particular, our experiments show consistent and reproducible values of $d_{c}$ even with several differing tips, which also confirms that the tip apex is reasonably smooth (see Appendix D for further discussions).

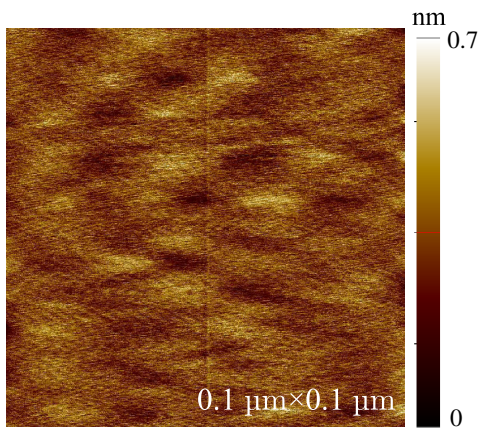

FIG. 6. Noncontact mode AFM scan image of the side surface of a quartz tip. Commercial AFM was employed to obtain the AFM image. We find that the root-mean-square roughness of the quartz surface is about $0.1 \mathrm{~nm}$.

\section{Effect of surface charge}

It is known that there exists a high electric potential $(>10 \mathrm{~V})$ on the mica surface right after cleavage due to the charge imbalance of $K^{+}$ions [27]. However, this uneven charge distribution disappears in ambient conditions in about a few minutes at $20 \% \mathrm{RH}$ and for as long as 24 hours in vacuum. In the present experiment, this charge effect can be neglected because we stabilized the system in a closed chamber in ambient conditions for at least 3 hours to 1 day before starting each set of measurements. Furthermore, the time interval between each measurement was changed from several minutes to several hours. Independent of this time interval, we have obtained consistent values of $d_{c}$ for each set of 100 measurements.

\section{Dependence of tip's motion}

We also performed statistical analysis of other effects such as the tip's oscillation amplitude and the staying time $\left(\tau_{s}\right)$. As shown in Figs. 7(a) and 7(b), $d_{c}$ is independent of the tip's shear amplitude as well as the approaching velocity ( $=0.05 \mathrm{~nm} / \tau_{s}$ ) within $\pm 0.1 \mathrm{~nm}$ error. Wide and thin shape
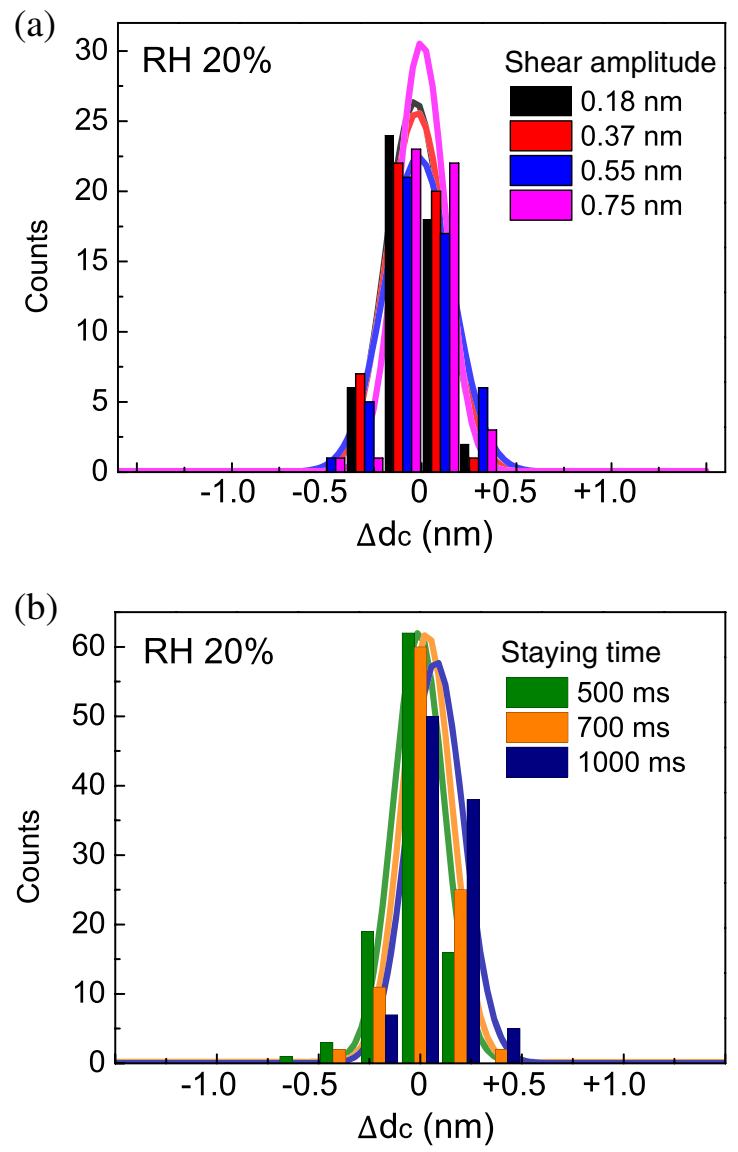

FIG. 7. (a) 50-times approach-retraction processes were conducted at differing shear amplitudes. (b) Similarly, 100-times processes were performed at various $\tau_{s}$. The histogram shows that $\mathrm{d}_{\mathrm{c}}$ does not depend on the amplitude nor approach the velocity $\left(=0.05 \mathrm{~nm} / \tau_{s}\right)$ within the available range. 
of the meniscus implies that a small lateral modulation of the tip provides negligible perturbation to the meniscus during precise control of the tip-surface distance, which indicates the meniscus stays in thermodynamic equilibrium, as also confirmed by our previous result that the oscillating tip did not appreciably perturb the nucleation rates [36].

\section{CURVATURE DEPENDENCE OF SURFACE TENSION AND TOLMAN LENGTH OF WATER}

Let us now compare our experimental results with the Kelvin equation. To do that, we need a relation between $R$ and $\mathrm{d}_{\mathrm{c}}$, which can be calculated by the Young-Laplace equation [37]. A simple estimate leads to $\mathrm{d}_{\mathrm{c}} \approx 2\left|r_{1}\right| \approx$ $0.46 \mathrm{~nm}$ at $10 \% \mathrm{RH}$. With $1 / r_{2}$ included, we find $\mathrm{d}_{\mathrm{c}} \approx$ $0.6 \mathrm{~nm}$ [blue curve, inset of Figs. 8(a), and 8(b); see
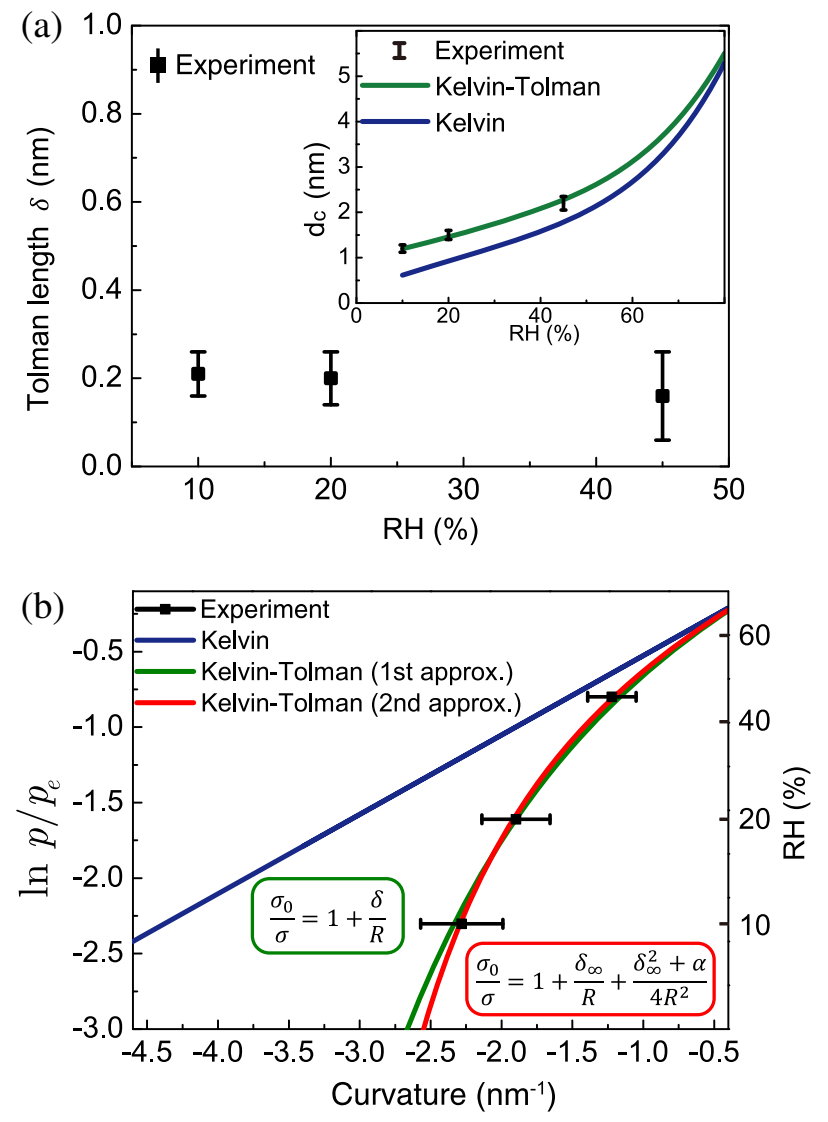

FIG. 8. (a) The Tolman length is derived for the measured $d_{c}$ at a given $\mathrm{RH}$ by fitting the equilibrium value of $1 / R$ in the first-order Kelvin-Tolman equation [Eq. (3)]. The inset shows the experimental $\mathrm{d}_{\mathrm{c}}$ and the theoretically calculated $\mathrm{d}_{\mathrm{c}}$ using the KelvinTolman equation ( $\delta=0.2 \mathrm{~nm}$, green curve) and the Kelvin equation with constant surface tension (blue curve). (b) Equilibrium curvature $(=1 / R)$ is calculated from the $\mathrm{d}_{\mathrm{c}}$ measurements. The green curve represents the first-order Kelvin-Tolman equation with $\delta=0.2 \mathrm{~nm}$ [same as in the inset of (a)], whereas the red curve is fitted on the second-order equation with $\delta_{\infty}=0.13 \mathrm{~nm}$ and $\delta_{\infty}^{2}+\alpha=-0.14 \mathrm{~nm}$, which are reasonably consistent with the homogeneous-nucleation measurements $[6,23]$.
Appendix $\mathrm{E}$ for calculation of $\mathrm{d}_{\mathrm{c}}$ ]. The apparent discrepancy with the measured $\mathrm{d}_{\mathrm{c}}$ of $1.2 \mathrm{~nm}$ at $10 \% \mathrm{RH}$ can be resolved when the curvature dependence of surface tension is considered, as given by the Tolman equation [17],

$$
\sigma=\frac{\sigma_{0}}{1+\frac{\delta}{R}}
$$

where $\sigma$ denotes the curvature-dependent surface tension, $\sigma_{0}$ is the surface tension of the planar interface, and $\delta$ is the Tolman length. Notice that although Eq. (2) was originally derived for a simple spherical shape of a liquid droplet, it has been shown that the same equation can be extended to a general shape of liquid beyond a sphere as shown in Ref. [38]. Combining Eqs. (1) and (2) gives the following general Kelvin-Tolman equation $[13,14]$ (see Appendix F):

$$
\frac{1}{R}=\frac{k_{B} T}{v_{l} \sigma_{0}}\left(1+\frac{\delta}{R}\right) \ln \frac{p}{p_{e}}
$$

which in fact corresponds to the first-order approximation of curvature dependence that assumes constant $\delta$.

For a qualitative discussion of Eq. (3), we first neglect the positive curvature $1 / r_{2}$ and find

$$
r_{1} \sim \frac{v_{l} \sigma_{0}}{k_{B} T} \frac{1}{\ln \frac{p}{p_{e}}}-\delta
$$

where the first term represents the Kelvin radius $(=-0.23 \mathrm{~nm}$ at $10 \% \mathrm{RH})$. This simple relation shows that the Tolman equation leads to a change in $r_{1}$ and thus a different $d_{c}$ value (Fig. 1). For a positive Tolman length, the curvature-dependent surface tension results in a larger absolute value of the radius of curvature, so capillary condensation occurs at a larger distance than the Kelvin equation predicts; however, for the negative sign, the opposite results occur. Notice that at high $\mathrm{RH}$, the Kelvin radius dominates, and thus the curvature effect is negligible [14].

For quantitative analysis, the exact shape of the meniscus should be calculated numerically by the Young-Laplace equation [37], from which fitting of Eq. (3) with respect to the measured $\mathrm{d}_{\mathrm{c}}$ produces the values of $\delta$ versus $\mathrm{RH}$; e.g., $\delta=0.21 \pm 0.05 \mathrm{~nm}$ at $10 \% \mathrm{RH}$ as shown in Fig. 8(a) [39]. The inset shows the theoretically calculated values of $\mathrm{d}_{\mathrm{c}}$ using the Kelvin-Tolman equation with a constant $\delta=0.2 \mathrm{~nm}$ (green curve) as well as the Kelvin equation (blue curve), respectively, under the same experimental constraints of tip radius and contact angles. As already discussed, the difference in $d_{c}$ between experimental measurement and prediction of the Kelvin equation is prominent, especially at low $\mathrm{RH}: 84 \%$ difference at $10 \%$ RH and $66 \%$ difference at $20 \%$ RH. Notice that as shown in Fig. 8(a), the Tolman length $\delta$ decreases slightly with RH, which indicates the higher-order curvature effect in $\delta$. 
By applying our fitting process to the more detailed secondorder approximation of the Kelvin equation [23] as shown in Fig. 8(b), we find $\delta_{\infty}=0.13 \pm 0.10 \mathrm{~nm}$ and $\delta_{\infty}^{2}+\alpha=$ $-0.14 \pm 0.12 \mathrm{~nm}^{2}$ (see Appendix G).

The positive sign of the Tolman length we determined indicates that water with high negative (positive) curvature has surface tension higher (lower) than the bulk value, so nucleation of water molecules, or formation of the critical nucleus of water, is more prevalent than normally expected; in other words, water is more abundant in the microscopic world of nature than typically considered. It should be noted that although the Tolman equation has long been recognized [20,40], the magnitude and even the sign of the Tolman length have been rather controversial due to the lack of experimental results [18-22]. Therefore, it is the very purpose of the present work to obtain the experimental value of the Tolman length that is obtained by direct measurements of $d_{c}$ for the critical nucleus of capillary condensation. Moreover, since surface tension is the rare physical quantity that is commonly and widely used in various fields of science and engineering, quantitative characterization at molecular scale implies its immediate, far-reaching impact for a deeper understanding of nanoconfined liquids as well as a critical nucleus.

Notice that the Kelvin equation describes the nucleation process that determines the equilibrium curvature of the liquid-vapor interface, which requires much care for its application. As shown in Fig. 2(c), the meniscus ruptures at large distances above $d_{c}$ because the meniscus hardly evaporates during tip retraction; thus, hysteresis appears [41]. Unlike the rupture, however, the approach process can be approximated as equilibrium since it is heading toward the condensed phase [33,41], which justifies our $\mathrm{d}_{\mathrm{c}}$-measurement scheme during approach in comparison to the rupture distance or force measurements made during retraction [26,27]. Moreover, the water meniscus can be formed if the following conditions are met [4]: (i) thermodynamic condition required for the equilibrium curvature and (ii) the geometric boundary condition imposed by the solids via contact angles. Therefore, whenever there is a geometrical solution satisfying these conditions, liquid meniscus is always nucleated through capillary condensation $[3,42]$.

\section{SUMMARY AND CONCLUDING REMARKS}

In summary, we have experimentally demonstrated that the Kelvin equation is valid down to the $0.5-\mathrm{nm}$ scale, if and only if the curvature-dependent surface tension is incorporated. This means that, in reality, water can condense at a lower RH as well as in a wider gap than the Kelvin equation predicts, indicating that water can be more ubiquitous in nature than normally expected. In particular, we have shown that the curvature-dependent surface tension evidently dominates the Kelvin equation in the molecular scale compared to any other effects associated with the critical nucleus, and provided the first direct measurement of the Tolman length in the well controlled water meniscus.

Notice that it has been challenging to measure the Tolman length not only because it has a very small value but also because, in such a molecular scale, other physical quantities cannot be determined simultaneously in a single experiment and thus lead to large uncertainties of the Tolman length. Although our study does not fully address how and how much the physical properties of the nanomeniscus become different from the continuum model in detail, this interesting and important issue should be pursued in subsequent research. Nonetheless, the significance of our work is that, while most of the experiments on the Tolman length have been performed so far on the spherical shapes such as the grown droplet and bubble, we have realized that the Tolman length can be measured by capturing, for the first time, the critical moment of nucleation for the meniscus, which may provide more direct and detailed information on the critical nucleus. We emphasize that the consistency of nucleation experiments in various geometries will therefore advance our knowledge of nucleation and also ultimately contribute to a molecular understanding of surface tension.

As the Kelvin equation is a basis for all nucleation phenomena, our findings provide a general understanding of homogeneous and heterogeneous nucleation at molecular scale. In particular, the fact that the surface tension can be investigated experimentally up to the second order of the curvature [red curve, Fig. 8(b)] will help guide further quantitative theoretical developments. This is important because the small curvature effect of surface tension $(\sim 10 \%)$ results in a significant change of the homogeneous nucleation rate by as much as 5 orders of magnitude [23], which justifies our work in that more accurate information of the critical nucleus provides a better understanding and control of nucleation.

Interestingly, the demonstrated curvature dependence of surface tension may account for the slightly smaller values of the activation diameter of aerosol particles in cloud formation below what the heterogeneous nucleation theory, based on the Kelvin equation, predicts [43]. Moreover, our findings suggest an alternative mechanism for how insoluble particles, especially organic particles, act as cloud condensation nuclei in atmospheric cloud formation [44,45]: Since capillary condensation between two hydrophilic surfaces can easily occur even at RH well below 100\%, two seed particles (or aerosol particles) may cooperatively act as cloud nuclei $[11,46,47]$, in addition to the well-known contribution of a single solid surface to cloud formation [5]. Therefore, for a unified understanding of particle formation and droplet activation, nucleation between two particles with curvature-dependent surface tension should be considered beyond other effects such as adsorption isotherm, collision rate, and chemical reactions $[48,49]$. 


\section{ACKNOWLEDGMENTS}

The authors acknowledge Professor Dongha Shin for helpful discussions and Brendan Deveney for critical reading of the manuscript. The SEM images of the quartz tip were obtained at the Research Institute of Advanced Materials, Seoul National University. This work was supported by the National Research Foundation of Korea (NRF) grant funded by the Korean government (MSIP) (No. 2016R1A3B1908660) and Basic Science Research Program through the NRF funded by the Ministry of Education, Science and Technology (No. 2017R1A6A3A11033301).

S. K. and D. K. contributed equally to this work.

\section{APPENDIX A: INVARIANT TIP SHAPE BEFORE AND AFTER MEASUREMENTS}

The SEM images of the quartz tip taken before and after the dc measurements (Fig. 9) show that the radius of curvature of the tip is approximately the same as about $40 \mathrm{~nm}$.

\section{APPENDIX B: STATISTICAL MEASUREMENTS OF $d_{c}$ WITHOUT COALESCENCE-INDUCED SHIFT}

We provide detailed processes of $d_{c}$ measurements that are presented in Figs. 2-4. At 10\% RH, the critical distance $\left(\mathrm{d}_{\mathrm{c}}\right)$ is measured constant as $1.2 \pm 0.1 \mathrm{~nm}$ averaged over two independent sets of 100-times repeated measurements on different mica samples using different tips but with a similar tip radius (notice that we also make a few other additional sets of 3 to 4 measurements at $10 \% \mathrm{RH}$ to determine the contact point using other mica samples and similar tips, which still provide the same $d_{c}$ ). This case is consistent with the previous study [29], which shows that at low RH, there is no or only partially distributed clusters of water on the mica.

At both $20 \%$ and $45 \% \mathrm{RH}$, on the other hand, we measure two distinct values of $d_{c}$, which is attributed to the presence of liquid film at the measurement position, obtained for different

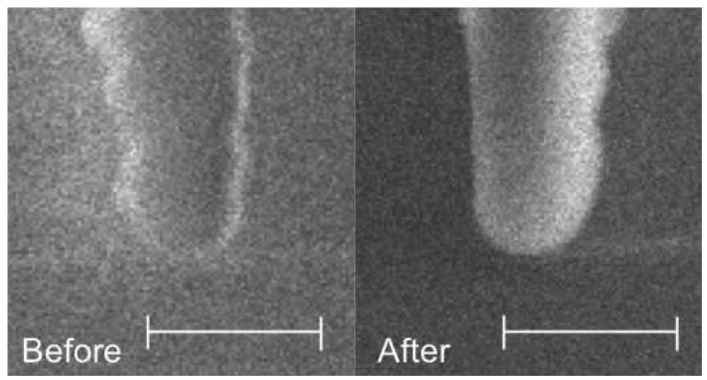

FIG. 9. The SEM image of a quartz tip shows that the tip shape and its radius of curvature $(\sim 40 \mathrm{~nm})$ do not change much before and after the experiments of more than 100 approach-retraction processes. The scale bar is $200 \mathrm{~nm}$. mica samples and tips having a similar tip radius. At $20 \% \mathrm{RH}$, in one set of 100 measurements, all the $d_{c}$ data belong to the average $1.5 \mathrm{~nm}$ [Fig. 2(c)], whereas in another set of similar 100 measurements made on a newly cleaved mica using another tip, all the $\mathrm{d}_{\mathrm{c}}$ values appear at the average $1.9 \mathrm{~nm}$ [Fig. 4(c)] [notice that subsequent measurements to check the systematic effects of shear-amplitude dependence and approach-speed dependence still produce the average $d_{c}$ of $1.9 \mathrm{~nm}$-Figs. 7(a) and 7(b)].

At $45 \% \mathrm{RH}$, two distinct values of $\mathrm{d}_{\mathrm{c}}$ are measured at 2.2 and $2.6 \mathrm{~nm}$ [Fig. 4(c)], obtained by two independent sets of 100 measurements using two mica samples and two similar tips (in addition, we also perform another third set of 100 measurements on a different mica but using the same tip as in the preceding experiment that produced $\mathrm{d}_{\mathrm{c}}$ of $2.6 \mathrm{~nm}$ at $45 \% \mathrm{RH}$, and obtain the average $\mathrm{d}_{\mathrm{c}}$ value of $2.2 \mathrm{~nm}$ ). Notice that each successive approach-retraction process is conducted under an interval of 5 minutes to several hours in order to avoid any possible effect of water that may remain on the mica after each rupture [27], while the tip is retracted by at least $100 \mathrm{~nm}$ before reapproaching the surface.

\section{APPENDIX C: RINSING MICA WITH ACID SOLUTION}

We perform supplementary experiments that compare two mica surfaces that were prepared by two different methods: tape cleaving and rinsing with dilute aqueous solution with the same tip (Fig. 10). For the rinsing method, we rinse the mica surface with $\mathrm{HCl}(\mathrm{pH} \sim 3)$ solution for 2 minutes and then dry with a flow of nitrogen gas, which is the same method as the one used in Ref. [14]. We find that the water film spreads out almost all over the rinsed surface, perhaps with different heights at different locations, even after drying with nitrogen gas for 20 minutes, and the resulting measured $d_{c}$ values and the associated uncertainties substantially increase by about $0.3 \mathrm{~nm}$ to $1 \mathrm{~nm}$. In addition, numerous approach processes are performed on the rinsed-mica surface, and a large distribution of $d_{c}$ values is observed; however, capillary condensation above the tape-cleaved mica surface produces the reproducible and considerably consistent $d_{c}$ values. We present some of the typical approach-retraction curves in Fig. 10, which confirms that the tape-cleaving method is indeed a reliable way for $d_{c}$ measurement to test the validity of the Kelvin equation without the water-film contribution.

For comparison, we have also conducted another experiment where the mica was rinsed with acid solution for 5 to 10 minutes and then with deionized water of more than $30 \mathrm{~mL}$, and then dried with nitrogen gas for 20 minutes. The results show behaviors similar to those obtained for mica that was rinsed only with acid solution without deionized water; e.g., the $d_{c}$ values increased with large variations $(\sim 1 \mathrm{~nm})$ depending on the location, and the meniscus ruptured at a large distance compared to the one obtained by pure tape cleaving of mica. 


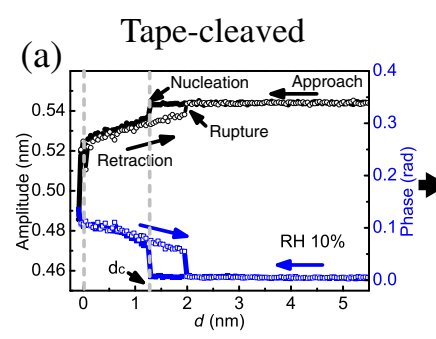

Rinsed with acid

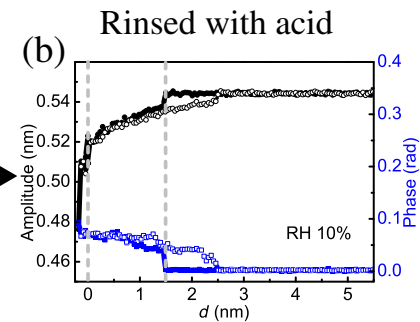

(c)

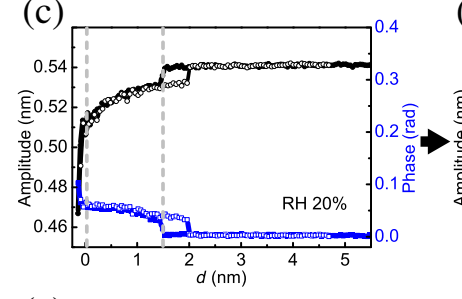

(d)

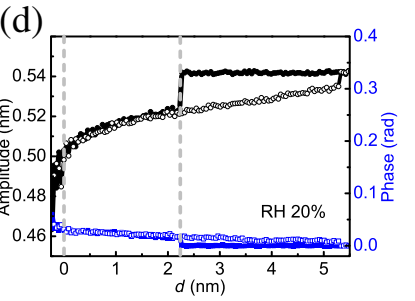

(e)

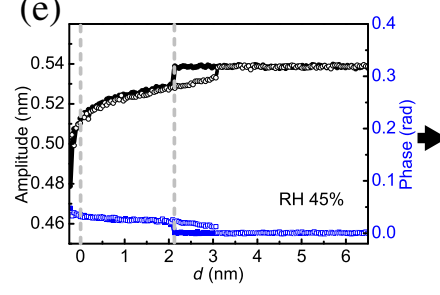

(f)

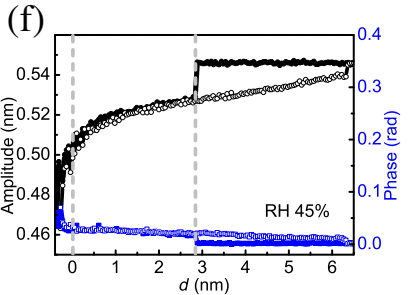

FIG. 10. Comparison of the critical distance depending on the mica preparation method. Panels (a), (c), and (e) are the typical approach-retraction curves above the tape-cleaved mica surface at differing RH. The corresponding panels [(b), (d), and (f)] are the approach-retraction curves on the mica that was rinsed with dilute aqueous solutions of $\mathrm{HCl}(\mathrm{pH} \sim 3$ ) for 2 minutes and then dried with a stream of nitrogen gas as in Ref. [14]. The same tip was used in both cases. As is clear, capillary condensation above the rinsed mica surface clearly shows larger critical distances.

\section{APPENDIX D: ROUGHNESS OF QUARTZ TIP}

In addition to the roughness measurement of the quartz tip by a commercial AFM (Fig. 6), we have investigated the effect of a rough tip on capillary condensation of the meniscus, which shows that only the smooth tip provides reliable $d_{c}$ measurements. By performing numerous approach-retraction processes using the tips with various radius of curvature and shapes, we find that when the tip apex is rough, there are enhanced signal fluctuations as the tip contacts the substrate as well as while the tip retracts until the meniscus ruptures (Fig. 11). This indicates that the approach-retraction curves indeed reflect the roughness of the tip apex; therefore, the clean approach-retraction curves that we obtained for $d_{c}$ measurements confirm that the tip apex is rather smooth. Notice that such a noisy signal is significantly reduced after repetitive hard contact of the tip on the substrate. This result suggests that the fluctuating signal results from the pinning-depinning effect and the multiple contact of the quartz tip with the surface (in the experiments described in our paper, we do not observe such fluctuations during 100-times approach-retraction sets of measurement). Notice also that whereas there are not

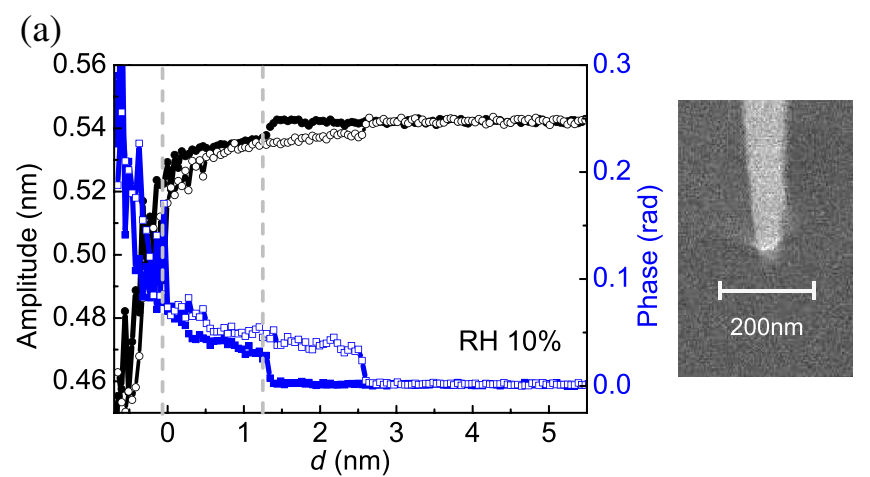

(b)

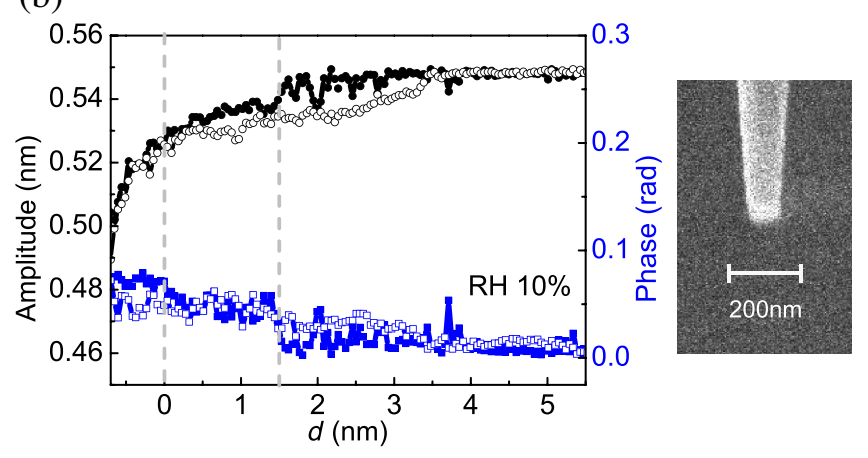

FIG. 11. Fluctuating amplitude and phase signals due to the rough-end tip during its contact with the substrate and subsequent retraction. Panels (a) and (b) represent separate experiments using different rough tips. Both experiments were conducted at $\mathrm{RH}$ $10 \%$. The SEM image of the tip is shown on the right side of each panel. The results indicate that the roughness of the tip apex is indeed reflected on the enhanced fluctuations in the approachretraction curves, and the fact that we have obtained clean approach-retraction curves suggests that the tip apex was not rough for our measurements.

noticeable differences in the measured values of $d_{c}$ even after we gently contact the smooth tip on the surface, large variations of $d_{c}$ are observed when a rough tip is used. Such consistent and reproducible results of $\mathrm{d}_{\mathrm{c}}$ measurement have also been demonstrated by performing additional supportive experiments using other well-prepared smooth tips, which are not presented here because of their identical values.

\section{APPENDIX E: CALCULATION OF $d_{c}$ USING THE YOUNG-LAPLACE EQUATION}

We show how to calculate the critical distance $\left(d_{c}\right)$ from a given curvature using the Young-Laplace equation. The Young-Laplace equation is a second-order ordinary differential equation describing the pressure difference between two bulk phases with a constant mean radius of curvature. The following equation describes the surface of a constant mean curvature for the symmetrical $z$ axis (i.e., pendular ring), 


$$
\frac{1}{R}=\frac{d^{2} z / d r^{2}}{\left(1+(d z / d r)^{2}\right)^{3 / 2}}+\frac{d z / d r}{r\left(1+(d z / d r)^{2}\right)^{1 / 2}}
$$

where $r$ is the horizontal axis and $z$ is the vertical axis of the meniscus [37]. Given the tip-surface distance, we can find a solution of Eq. (E1) satisfying the boundary conditions, which is done by numerically solving the elliptic integrals. In other words, with the given curvature $1 / R$, we can find the exact $d_{c}$, above which there exists no solution.

\section{APPENDIX F: DERIVATION OF THE KELVIN-TOLMAN EQUATION}

Assuming the incompressibility of a liquid and the ideal gas law, the Kelvin equation is derived by the chemical potential change from a planar surface to a curved one as follows;

$$
\begin{gathered}
d \mu=-s d T+v d p, \\
\Delta \mu_{v}=\int_{p_{e}}^{p} \frac{k_{B} T}{p} d p=\Delta \mu_{l}=v_{l} \int_{p_{e}}^{p_{l}} d p, \\
p_{l}-p=\frac{\sigma}{R}, \\
v_{l}\left(\frac{\sigma}{R}+p-p_{e}\right)=k_{B} T \ln \frac{p}{p_{e}},
\end{gathered}
$$

where Eq. (F1) comes from the fundamental thermodynamic equation and $\mu_{v}, \mu_{l}$ are chemical potentials of vapor and liquid, respectively, $p$ is the external vapor pressure, and $p_{e}$ is the saturation pressure. Equation (F3) is the Young-Laplace equation. Other constants are described in the main text. Notice that the $\sigma / R$ term is much larger than $p-p_{e}$, which leads to the Kelvin equation [Eq. (1)].

The Young-Laplace equation can be derived from fundamental thermodynamics when the interfacial tension is defined at the surface of tension. Also, this interfacial tension defined at the surface of tension, which we call the surface tension, is a function of curvature. This is established by the Gibbs-Tolman-Koenig-Buff (GTKB) equation, which can be derived using the Gibbs adsorption equation and the Young-Laplace equation [50].

The Tolman length is then defined in terms of the superficial density divided by the density difference of two bulk phases, where the superficial density is defined as the difference between the actual amount of fluid particles and the amount of particles it would contain if the bulk phases retained a uniform density up to the surface of tension [17]. A more general approach to the Tolman equation, other than the simple spherical geometry, is provided in Ref. [38]. Since the Young-Laplace equation is defined at the surface of tension, $\sigma$ in Eq. (1) is also a function of curvature.

\section{APPENDIX G: SECOND-ORDER CURVATURE EFFECT IN THE TOLMAN EQUATION}

Recent work on the homogeneous nucleation (HON) theory has revealed that the curvature dependence of the Tolman length itself should be included in order to consistently account for the nucleation rate measured in the HON processes (i.e., the number of nucleation events per unit volume and time) [23,51,52]. This could be achieved by Taylor expanding and integrating the original GTKB equation. The curvature dependence of the Tolman length is then approximated as follows [51]:

$$
\delta\left(\frac{1}{R}\right)=\delta_{\infty}+\frac{\alpha}{2 R}
$$

where $1 / R$ is twice the mean curvature $\left(=2 / R_{S}\right.$ in the case of a sphere of radius $R_{s}$ ) defined on the surface of tension, $\delta_{\infty}$ is the curvature-independent term, and $\alpha$ is the coefficient of the second term. This gives the curvature dependence of surface tension as follows:

$$
\frac{\sigma_{0}}{\sigma}=1+\frac{\delta_{\infty}}{R}+\frac{\left(\delta_{\infty}^{2}+\alpha\right)}{4 R^{2}},
$$

where the higher-order $1 / R^{3}$ term beyond the second order is neglected in the Taylor expansion. Fitting Eq. (G2) to our experimental data now yields $\delta_{\infty}=0.13 \pm 0.10 \mathrm{~nm}$ and $\delta_{\infty}^{2}+\alpha=-0.14 \pm 0.12 \mathrm{~nm}^{2}$, which are in good agreement with the results of HON [6,23] (see Fig. S4 of Ref. [23]). Interestingly, this is an important and surprising observation, which consistently describes the two seemingly unrelated fields of research: AFM (heterogeneous nucleation) experiments and homogeneous nucleation rate experiments.

[1] R. Zhang, Getting to the Critical Nucleus of Aerosol Formation, Science 328, 1366 (2010).

[2] M. Kulmala, J. Kontkanen, H. Junninen, K. Lehtipalo, H. E. Manninen, T. Nieminen, T. Petäjä, M. Sipilä, S. Schobesberger, P. Rantala et al., Direct Observations of Atmospheric Aerosol Nucleation, Science 339, 943 (2013).

[3] M. Yarom and A. Marmur, Vapor-Liquid Nucleation: The Solid Touch, Adv. Colloid Interface Sci. 222, 743 (2015).

[4] J. N. Israelachvili, Intermolecular and Surface Forces (Academic Press, New York, 2011).

[5] D. Kashchiev, Nucleation (Elsevier, New York, 2000).

[6] D. Brus, V. Ždímal, and J. Smolík, Homogeneous Nucleation Rate Measurements in Supersaturated Water Vapor, J. Chem. Phys. 129, 174501 (2008).

[7] R. Evans, U. M. B. Marconi, and P. Tarazona, Capillary Condensation and Adsorption in Cylindrical and Slit-Like Pores, J. Chem. Soc., Faraday Trans. 2 82, 1763 (1986).

[8] M. Binggeli and C. Mate, Influence of Capillary Condensation of Water on Nanotribology Studied by Force Microscopy, Appl. Phys. Lett. 65, 415 (1994). 
[9] E. Riedo, F. Lévy, and H. Brune, Kinetics of Capillary Condensation in Nanoscopic Sliding Friction, Phys. Rev. Lett. 88, 185505 (2002).

[10] M. Lee, B. Kim, J. Kim, and W. Jhe, Noncontact Friction via Capillary Shear Interaction at Nanoscale, Nat. Commun. 6, 7359 (2015).

[11] Q. Guo, Y. Liu, G. Jiang, and X. Zhang, Cooperative Effect in Nucleation: Nanosized Seed Particles Jointly Nucleate Sapor-Liquid Transitions, J. Chem. Phys. 138, 214701 (2013).

[12] L. Bocquet, E. Charlaix, S. Ciliberto, and J. Crassous, Moisture-Induced Ageing in Granular Media and the Kinetics of Capillary Condensation, Nature (London) 396, 735 (1998).

[13] L. R. Fisher and J. N. Israelachvili, Direct Experimental Verification of the Kelvin Equation for Capillary Condensation, Nature (London) 277, 548 (1979).

[14] M. M. Kohonen and H. K. Christenson, Capillary Condensation of Water between Rinsed Mica Surfaces, Langmuir 16, 7285 (2000).

[15] M. H. Factorovich, V. Molinero, and D. A. Scherlis, Vapor Pressure of Water Nanodroplets, J. Am. Chem. Soc. 136, 4508 (2014).

[16] J. C. Melrose, Model Calculations for Capillary Condensation, AlChE J. 12, 986 (1966).

[17] R. C. Tolman, The Effect of Droplet Size on Surface Tension, J. Chem. Phys. 17, 333 (1949).

[18] Y. A. Lei, T. Bykov, S. Yoo, and X. C. Zeng, The Tolman Length: Is It Positive or Negative?, J. Am. Chem. Soc. 127, 15346 (2005).

[19] A. E. Van Giessen and E. M. Blokhuis, Determination of Curvature Corrections to the Surface Tension of a LiquidVapor Interface through Molecular Dynamics Simulations, J. Chem. Phys. 116, 302 (2002).

[20] M. J. P. Nijmeijer, C. Bruin, A. B. Van Woerkom, A. F. Bakker, and J. M. J. Van Leeuwen,Molecular Dynamics of the Surface Tension of a Drop, J. Chem. Phys. 96, 565 (1992).

[21] A. Tröster and K. Binder, Positive Tolman Length in a Lattice Gas with Three-Body Interactions, Phys. Rev. Lett. 107, 265701 (2011).

[22] M. A. Anisimov, Divergence of Tolman's Length for a Droplet near the Critical Point, Phys. Rev. Lett. 98, 035702 (2007).

[23] N. Bruot and F. Caupin, Curvature Dependence of the Liquid-Vapor Surface Tension Beyond the Tolman Approximation, Phys. Rev. Lett. 116, 056102 (2016).

[24] M. Lee, J. Jahng, K. Kim, and W. Jhe, Quantitative Atomic Force Measurement with a Quartz Tuning Fork, Appl. Phys. Lett. 91, 023117 (2007).

[25] B. Kim, S. Kwon, M. Lee, Q. Kim, S. An, and W. Jhe, Probing Nonlinear Rheology Layer-by-Layer in Interfacial Hydration Water, Proc. Natl. Acad. Sci. U.S.A. 112, 15619 (2015).

[26] L. R. Fisher and J. N. Israelachvili, Determination of the Capillary Pressure in Menisci of Molecular Dimensions, Chem. Phys. Lett. 76, 325 (1980).

[27] L. Xu, A. Lio, J. Hu, D. Ogletree, and M. Salmeron, Wetting and Capillary Phenomena of Water on Mica, J. Phys. Chem. B 102, 540 (1998).
[28] B. Kim, S. Kwon, H. Mun, S. An, and W. Jhe, Energy Dissipation of Nanoconfined Hydration Layer: Long-Range Hydration on the Hydrophilic Solid Surface, Sci. Rep. 4, 6499 (2014).

[29] J. Hu, X.-D. Xiao, D. F. Ogletree, and M. Salmeron, Imaging the Condensation and Evaporation of Molecularly Thin Films of Water with Nanometer Resolution, Science 268, 267 (1995).

[30] T. Arai, K. Sato, A. Iida, and M. Tomitori, Quasi-Stabilized Hydration Layers on Muscovite Mica under a Thin Water Film Grown from Humid Air, Sci. Rep. 7, 4054 (2017).

[31] J. N. Israelachvili, N. A. Alcantar, N. Maeda, T. E. Mates, and M. Ruths, Preparing Contamination-Free Mica Substrates for Surface Characterization, Force Measurements, and Imaging, Langmuir 20, 3616 (2004).

[32] J. C. Melrose, Chemical Potential Changes in Capillary Condensation, J. Colloid Interface Sci. 38, 312 (1972).

[33] J. Jang, G. C. Schatz, and M. A. Ratner, Liquid Meniscus Condensation in Dip-Pen Nanolithography, J. Chem. Phys. 116, 3875 (2002).

[34] N. Maeda, J. N. Israelachvili, and M. M. Kohonen, Evaporation and Instabilities of Microscopic Capillary Bridges, Proc. Natl. Acad. Sci. U.S.A. 100, 803 (2003).

[35] G. J. Simpson, D. L. Sedin, and K. L. Rowlen, Surface Roughness by Contact versus Tapping Mode Atomic Force Microscopy, Langmuir 15, 1429 (1999).

[36] B. Sung, J. Kim, C. Stambaugh, S.-J. Chang, and W. Jhe, Direct Measurement of Activation Time and Nucleation Rate in Capillary-Condensed Water Nanomeniscus, Appl. Phys. Lett. 103, 213107 (2013).

[37] F. M. Orr, L. E. Scriven, and A. P. Rivas, Pendular Rings between Solids: Meniscus Properties and Capillary Force, J. Fluid Mech. 67, 723 (1975).

[38] J. C. Melrose, Thermodynamic Aspects of Capillarity, Ind. Eng. Chem. 60, 53 (1968).

[39] D. Kashchiev, Determining the Curvature Dependence of Surface Tension, J. Chem. Phys. 118, 9081 (2003).

[40] D. Choi, M. S. Jhon, and H. Eyring, Curvature Dependence of the Surface Tension and the Theory of Solubility, J. Chem. Phys. 53, 2608 (1970).

[41] Y. Men, X. Zhang, and W. Wang, Capillary Liquid Bridges in Atomic Force Microscopy: Formation, Rupture, and Hysteresis, J. Chem. Phys. 131, 184702 (2009).

[42] F. Restagno, L. Bocquet, and T. Biben, Metastability and Nucleation in Capillary Condensation, Phys. Rev. Lett. 84, 2433 (2000).

[43] P. M. Winkler, G. Steiner, A. Vrtala, H. Vehkamäki, M. Noppel, K. E. J. Lehtinen, G. P. Reischl, P. E. Wagner, and M. Kulmala, Heterogeneous Nucleation Experiments Bridging the Scale from Molecular Ion Clusters to Nanoparticles, Science 319, 1374 (2008).

[44] M. O. Andreae and D. Rosenfeld, Aerosol-Cloud-Precipitation Interactions. Part 1. The Nature and Sources of Cloud-Active Aerosols, Earth-Sci. Rev. 89, 13 (2008).

[45] R. Sorjamaa and A. Laaksonen, The Effect of $\mathrm{H}_{2} \mathrm{O}$ Adsorption on Cloud Drop Activation of Insoluble Particles: A Theoretical Framework, Atmos. Chem. Phys. 7, 6175 (2007).

[46] Y. Crouzet and W. H. Marlow, Calculations of the Equilibrium Vapor Pressure of Water over Adhering 50-200-nm Spheres, Aerosol Sci. Technol. 22, 43 (1995). 
[47] S. Sjogren, M. Gysel, E. Weingartner, U. Baltensperger, M. J. Cubison, H. Coe, A. A. Zardini, C. Marcolli, U. K. Krieger, and T. Peter, Hygroscopic Growth and Water Uptake Kinetics of Two-Phase Aerosol Particles Consisting of Ammonium Sulfate, Adipic and Humic Acid Mixtures, J. Aerosol Sci. 38, 157 (2007).

[48] W. H. Marlow, Derivation of Aerosol Collision Rates for Singular Attractive Contact Potentials, J. Chem. Phys. 73, 6284 (1980).

[49] J. Ovadnevaite, A. Zuend, A. Laaksonen, K. J. Sanchez, G. Roberts, D. Ceburnis, S. Decesari, M. Rinaldi, N. Hodas, M. C. Facchini et al., Surface Tension Prevails over Solute
Effect in Organic-Influenced Cloud Droplet Activation, Nature (London) 546, 637 (2017).

[50] R. C. Tolman, Consideration of the Gibbs Theory of Surface Tension, J. Chem. Phys. 16, 758 (1948).

[51] V. G. Baidakov and K. S. Bobrov, Spontaneous Cavitation in a Lennard-Jones Liquid at Negative Pressures, J. Chem. Phys. 140, 184506 (2014).

[52] A. Tröster, M. Oettel, B. Block, P. Virnau, and K. Binder, Numerical Approaches to Determine the Interface Tension of Curved Interfaces from Free Energy Calculations, J. Chem. Phys. 136, 064709 (2012). 